\title{
Banja de Majakovskij entre profane et sacré.
}

Coïncidence ? La pièce Banja est créée au théâtre Mejerkhol'd le 16 mars 1930. Son auteur se suicide le 14 avril 1930, moins d'un mois après. Le 3 mars le critique influent Ermilov, sur la base d'une lecture de la pièce, démolit méthodiquement cette œuvre. Publié dans « La Sentinelle littéraire »1, organe de la RAPP (Association russe des écrivains prolétaires) dont l'autorité était contestée par Majakovskij, l'article de Ermilov fustige le « comportement gauchiste et petit-bourgeois $\left.{ }^{2}{ }\right\rangle$ de l'auteur. Pour faire bonne mesure la Pravda reprend cet article qu'il publie une semaine plus tard. Comment se leurrer ? En critiquant Pobedonosikov Majakovskij attaque de front la bureaucratie soviétique et donc le parti tout entier. Ermilov conseille à Majakovskij de parler de la vie présente au lieu de se transporter dans le futur. Dans le cadre de la lutte intense qui agite les milieux littéraires, cet article est une exécution en règle d'un écrivain épris de liberté.

La pièce Banja, comporte plusieurs niveaux qui servent à cacher sa signification profonde. On peut considérer qu'il s'agit du testament de l'auteur. La pièce est doublement cryptée. D'une part, la bouffonnerie. D'autre part, la satire. Derrière la bouffonnerie et la satire se cache un être écorché, un homme tout simplement qui se confronte à sa fin et à la fin du rêve de libération de son pays.

Déjà sa pièce Mystère-Bouffe présentait une telle multiplicité de sens. Il y avait le jeu grotesque des purs et des impurs, représentation imagée de la révolution. Mais à côté du Bouffe, il y avait le Mystère. Majakovskij se rattache à la tradition des Mystères du Moyen Age et met en scène le Christ, mais un Christ qui loin de proclamer la soumission appelle les croyants à la révolte.

Le mystère se retrouve dans Banja. Inspiré par Khlebnikov qu'il admirait, Majakovskij est obsédé par la fuite des temps, par l'éternité qui nous échappe et que le poète veut prendre dans ses rets. L'axe de la pièce est le temps, la machine du temps, la ma-

\footnotetext{
1 На литературном посту.
}

2 В.В. Ермилов, «О настроениях мелкобуржуазной «левизны» в художественной литературе», Правда,, 9 mars 1930.. 
chine à vaincre le temps, c'est une apothéose du temps et en même temps une victoire sur le temps.

La machine inventée par Cudakov permet de réaliser ce rêve de l'humanité, la maîtrise du temps. Avec elle on pénètre dans le futur. Bien sûr Herbert George Wells avait déjà imaginé ce saut dans une dimension interdite à l'homme. Mais ici c'est vraiment l'apocalypse de notre temps. Tiens, tiens, cela rappelle un certain Rozanov, un rebelle lui aussi.

Le temps fait l'objet de jeux de mots : « Temps, en avant ! », « Il faut lui couper son temps de parole », « Nous allons déchirer le temps décrépit ». Le temps est une composante de la vitesse et fait partie intégrante de véhicules comme le tramway et le train dont Pobedonosikov est un grand amateur. Il prépare un discours édifiant sur le tramway soviétique, tandis que le train éveille en lui la nostalgie d'un voyage d'agrément dans le Midi. Mais il y a, bien sûr, la machine du temps qui est elle-même un véhicule, une sorte de fusée. Comme on sait, les personnages principaux de la pièce, les bureaucrates soviétiques (Pobedonosikov, Optimistenko) et les opportunistes à leur service (Belvedonskij, Mesaliansova, Momental'nikov) ne sont pas admis dans le futur. La conclusion à la Gogol est mise dans la bouche de Pobedonosikov qui se tourne vers le public: «Vous voulez dire que les gens comme moi ne sont pas faits pour le socialisme ${ }^{3}{ }^{\text {» }}$

S'agit-il simplement d'une revendication politique ? Avec la machine du temps, machine invisible, l'auteur met le présent à la merci du futur, le présent est jugé par le futur. C'est le destin de l'homme qui est en jeu. L'homme, la société russe sont confrontés à un événement capital, le jugement dernier. La Femme phosphorescente, qui provient de l'année 2030, et qui fait le tri entre les bons et les mauvais, est une figuration de la Femme couronnée d'étoiles de l'Apocalypse. Comment dans l'Apocalypse les bons sont d'un côté, les méchants de l'autre. Qui sont les bons qu'elle emporte dans le futur ? Ce sont ceux qui sont prêts à s'intégrer à la commune. « L'avenir acceptera tous ceux qui manifesteront ne serait-ce qu'un minimum d'appartenance au collectif de la com-

\footnotetext{
3 Vladimir Majakovskij, Banja, Tome 4, Acte 6, «Stikhi, poemy, piesy », Khudožestvennaja literatura, M . 1936, p. 355.
} 
mune ${ }^{4}$. Faut-il voir dans la commune un synonyme de communisme ? On peut se demander si Majakovskij ne pense pas plutôt à cette utopie imaginée par Fiodorov sous le nom de « Cause commune ${ }^{5}$ », ce rêve immense d'une humanité régénérée, capable de faire revivre ses ancêtres ${ }^{6}$.

Les qualités exigées des bons sont assez curieusement celles que promeut le christianisme: « Joie de travailler, joie de se sacrifier, volonté d'inventer, joie de donner, fierté d'être un homme (...) Le temps qui fuit balaiera le ballast des hommes desséchés par manque de foi. ${ }^{7}$ »

La joie de donner. On pense à ces vers de Khlebnikov dans son poème de 1921, Trompettez, criez, portez ${ }^{8}$ !

Sachez, la Volga souffre de sécheresse,

Cela doit suffire pour ne pas prendre, mais pour donner.9

Roman Jakobson est frappé par l'accent khlebnikovien de la première réplique de Баня qui est précisément consacrée à la victoire sur le temps. ${ }^{10}$

Cudakov : La Volga du temps humain, où nous étions jetés jusqu'à présent par notre naissance pour partir à la dérive, ballottés par les vagues, cette Volga-là nous est

\footnotetext{
${ }^{4}$ Vladimir Majakovskij, Banja, Tome 4, 1er Acte, op.cit., Tome 4, p. 280.

Будущее примет всех, у кого найдется хотя бы одна черта, роднящая с коллективом коммуны.

5 общее дело

6 Semënova. S. G., Filosof budušcego veka Nilolaj Fëdorov, M. 2004

${ }^{7}$ Vladimir Maïakovskij, Banja, op.cit, Acte 6, Tome 4, p. 353.

Радость работать,жажда жертвовать, неутомимость исзобретать, выгода отдавать, гордость человечестью (...) Летящее время сметет и стрежет баласт, отягченный хламом, баласт опустошенных неверием.»

8 « Трубите, кричите, несите! »

9 Но знайте - На Вомге засуча: :

ЕАинственный повоА, чтобы не взять,а -дать»'

Khlebnikov, Stikhotvoreniia i poemy, Sovietski pisatel', Leningrad, 1960, p.168

${ }^{10}$ Roman Jakobson, recueil, Smert' Majakovskogo, Berlin , 1931, p.27. Observation tirée de la remarquable étude de R.V. Duganov,, Zamysel « Bani » Majakovskogo, www.ka2.ru.
} 
soumise. Selon mon bon plaisir le temps arrêtera son cours ou fuira dans la direction désignée à la vitesse voulue. Les hommes pourront quitter les jours comme ils quittent un tram ou un autobus. Ma machine te permettra d'immobiliser un instant de bonheur et de t'en saouler tant que tu en auras envie.»11

Comparons avec le poème Les tablettes du destin écrit par Khlebnikov peu de temps avant sa mort :

Le jour où l'on a pris la dimension du cours de la Volga est devenu le jour de sa soumission sous le joug de la voile et de la rame, de la reddition de la Volga à l'homme... Exemples semblables pour le cours du temps, établissant les lois du jour du lendemain, étudiant le cours des temps à venir....C'est depuis longtemps un lieu commun que connaître c'est pouvoir, et que prévoir les événements c'est s'en rendre maîtres. ${ }^{12}$

Il n'est pas jusqu'à l'instrument typique du temps, la montre, qui ne montre une parenté entre Majakovskij et Khlebnikov.

Tchoudakov. Vos montres, vous pouvez-les donner en gage et les vendre. Velossipedkine. Heureusement que je n'en ai pas encore acheté! Tchoudakov. N'achète pas de montre. N'en achète surtout pas! Bientôt cette bêtise toute plate et faisant tictac sera plus ridicule qu'une bougie dans une centrale électrique, plus inutile qu'un taureau sur l'autoroute. ${ }^{13}$ »

Chez Khlebnikov la montre joue aussi un rôle capital dans le destin de l'humanité :

Si je transforme l'humanité en montre Et démontre comment avance l'aiguille des siècles, Est-ce que votre ruban des temps

Ne donnera pas son envol à la guerre comme à une voyelle inutile ? (...)

11 Vladimir Maïakovski,j Théâtre, Fasquelle, Paris, 1972, trad. Wassiltchikov, p.234..

12 Velimir Khlebnikov, Doski sud'by, List 1, « Promery sud'by », in Babkov Vassili, Konteksty Dosok Sud'by, M. 2000.

13 Vladimir Majakovskij, Banja, op.cit. Tome 4, 1er Acte, , p. 280. (traduit par moi, GA). 
Montre de l'humanité, avec ton tic-tac

Fais avancer mes pensées avec le mouvement de tes aiguilles. ${ }^{14}$

Le tic-tac de la montre se retrouve chez l'un et chez l'autre, même si chez Majakovskij le ton soit celui de la plaisanterie.

Dans son récit « Ka » Khlebnikov rencontre un peintre (Filonov ?):

Je lui demandai s'il partirait à la guerre. Il répondit : « Moi aussi je mène une guerre, seulement ce n'est pas une guerre pour l'espace, mais pour le temps. Je demeure dans une tranchée et j'arrache au passé une parcelle du temps. Mon devoir est aussi pénible à accomplir que celui des troupes qui se battent pour l'espace 15

... Si le temps acquiert une signification métaphysique qu'en est-il de la Баня ? Pour faire diversion Majakovskij déclare que c'est une manière de laver la Russie du chancre de la bureaucratie : « Les Bains servent tout simplement à laver, à lessiver les bureaucrates $»^{\mathbf{1 6}}$. Fonction purement prophylactique dans le domaine social et politique. Mais il fait par ailleurs une révélation capitale:

Pourquoi banja? Parce que c'est la seule chose qui ne se trouve pas dans la pièce ${ }^{17}$.

C'est évidemment une manière d'esquiver la réponse, une boutade comme aimait à en faire Majakovskij . Le poète ouvre subrepticement une brèche dans sa carapace, révélant l'absence, le non-être, le néant existentiel qui nourrit son œuvre en profondeur.

14 Khlebnikov Velemir, Dosok sud'by, list 1, Zarej Vencannyj. 28-1-1922.

Если я обращу человечество в часы

И покажу, как стрелка столетий движется,

Неужели ив вашей времен полосы

Не вылетит война, как ненужная ижица? (...)

Часы человечества, тикая ,

Стрелкой моей мысли двигайте!

15 Khlebnikov, Nouvelles du Jeu et du monde, trad. JC Lanne, Paris, Imprimerie Nationale, 1994, p.223-224.

Я спросил, пойдет ли он на войну? Он ответил: “Я тоже веду войну, только не за пространство, а за время. Я сижу в окопе и отымаю у прошлого клочок времени. Мой долг одинаково тяжел, что и у войск за пространство".

16 « Баня” моет (просто стирает) бюрократов », Vladimir Majakovskij, Polnoe sbranie socinenij, Khudožestvennaja literatura, M. 1959. Tom XII, p. 200.

17 Vladimir Majakovskij, Ibid. Tom XII, p.379. Почему Баня? Потому, что это единственное,что там не попадается. 
Quel contraste avec le spectacle en six actes avec feux d'artifice qui se donne à voir au théâtre. La banja ne se réduit pas à sa fonction sanitaire. La banja est ancrée dans la culture archaïque du peuple russe. Dans la banja on se lave, certes, mais la purification opérée par l'eau est aussi purification de l’âme.

Depuis l'Antiquité et dans tout l'Orient les bains publics sont une institution inhérente à la vie commune, depuis les villes jusqu'au plus petit des villages. Dans le Nord de l'Europe il y a le sauna des Finlandais et la banja des Russes. Dans la banja on produit une vapeur d'eau bouillante en versant de l'eau sur des pierres chauffées à blanc. Ensuite on se fouette avec des branches de bouleau pour activer la circulation et on se refroidit à l'eau froide ou en se plongeant soit dans un cours d'au voisin, soit dans la neige.

Petite construction, en bois située à l'écart du village, lieu qu'il ne fait pas bon fréquenter à la tombée de la nuit, la banja est une sorte de templum, où l'on se dévêt complètement. L'homme s'y retrouve hors de l'espace, et hors du temps. Le temps qui est une obsession de la poésie khlebnikovienne est le personnage central de la pièce Banja.

La banja est le lieu où le temps de la fin fait irruption dans le moment présent. Cet espace sacré est en effet le lieu du mystère. Sacré et transgression. Le bannik se cache au fond de la banja pour déranger les baigneurs. Mais il a aussi un don de prophétie. Malgré le danger Les jeunes filles du village vont l'interroger la nuit pour savoir qui sera leur fiancé. Lieu de la purification la banja est un lieu d'accouchement, qui assure au nouveau-né la protection des forces sacrées. Mais on peut aussi mourir dans la banja. Ce fut le cas de Khlebnikov qui se fit transporter dans la banja de Santalovo, le village où il a été hébergé par le peintre Miturin. C'est là qu'il a choisi de mourir.

La banja est le lieu de la vie, c'est aussi le lieu de la mort. Pour Majakovskij il faut se ressaisir et dépasser l'idée d'un jugement dernier qui séparerait les bons des méchants. L'humanité doit se transformer pour accéder à la régénérescence promise par l'avenir dans une sorte d'apocalypse. Pour Dostoevskij la banja est plutôt une porte qui 
ouvre sur l'enfer. On se souvient des détenus du bagne d'Omsk, conduits par groupes de cinquante dans la banja de la ville où l'on est confronté à une vision dantesque .

Il me vint l'esprit que, si nous devions un jour nous retrouver tous ensemble dans [la fournaise de] l'enfer, cela rappellerait fort le lieu où nous nous trouvions. ${ }^{18}$

C'est ici que la traduction en français du mot banja conduit à des contresens, car il dénote une réalité qui n'évoque rien de précis dans notre civilisation. Suivant les cas on trouve: bains, étuve, grande lessive. Ce dernier terme qui a été choisi par un des traducteurs ${ }^{19}$ a l'inconvénient de réduire à une simple réalité prophylactique la dimension sacrée de la banja. On comprend le choix du traducteur de Dostoevskij qui s'affranchit du terme de bains pour adopter celui d'étuves ${ }^{20}$. Il traduit ainsi la forte chaleur humide qui règne dans le où l'on conduit les détenus, autant par hygiène que pour sacrifier à des rites ancestraux. De ce fait dans le cours de cette étude nous préférons conserver sans le traduire le terme si chargé de banja.

Sensible à l'ambiguiité inhérente à ce terme, Dostoevskij le dépouille de son caractère sacré dans un passage de Crime et châtiment où Svidrigajlov, sur le seuil de la mort qu'il va se donner, en donne une vision d'autant plus terrifiante qu'elle est d'un prosaïsme décapant. La banja est la métaphore du néant.

Raskolnikov : Je ne crois pas à la vie future.

Svidrigaïlov : Et s'il n'y avait là que des araignées ou autres bêtes semblables ? (...) Nous nous représentons toujours l'éternité comme une idée impossible à comprendre, quelque chose d'immense ! Mais pourquoi en serait-il nécessairement ainsi? Et si, au lieu de tout cela, il n'y a, figurez-vous, qu'une petite baraque, comme qui dirait une de ces [banja] de village, tout enfumée, avec des toiles d'araignées dans tous les coins : la voilà l'éternité! " ${ }^{21}$

\footnotetext{
18 Dostoievski, Souvenirs de la maison des morts, « La pléiade », Gallimard, Paris, trad. H. Mongault et L.Desormonts. p.1007.

Мне пришло на ум, что если все мы вместе будем когда-нибудь в пекле, то оно очень будет похоже на это место.

19 Vladimir Maïakovski, La Grande lessive, op.cit, p.221- 323.

20 Dostoievski, Souvenirs de la maison des morts, op.cit., p.1005. «Quand nous ouvrîmes la porte de l'étuve, je crus pénétrer en enfer ».

${ }^{21}$ Dostoievski Crime et châtiment, Paris, « La pléiade », Gallimard, 1950, trad. D. Ergaz, p.313. (Première édition en 1866)

J'ai rétabli « banja » au lieu de « cabines de bains » et « baraque » au lieu de « chambre ». GA
} 
Svidrigajlov veut ébranler les certitudes et l'orgueil de Raskol'nikov. Il dessine une réalité sordide et dépourvue de tout romantisme. Il se veut factuel, objectif, sans fioritures. Ces araignées repoussantes sont bien réelles, tapissant les murs de la banja mais ne sont-elles pas tapies au fond de nous-mêmes ? On se souviendra que quelques années avant la publication du roman de Dostoevskij, Baudelaire avait dénoncé l'emprise du spleen, dressant « au fond du cerveau » sa toile d'araignée.

[Et qu'] un peuple muet d'infâmes araignées

Vient tendre ses filets au fond de nos cerveaux ${ }^{22}$

La victoire de la Femme phosphorescente est la victoire de l'utopie. Qu'a-t-elle à offrir à ceux qui la suivent, sinon une sorte de fuite hors du réel, qui n'est pas salvatrice, elle non plus. Si les meilleurs s'en vont, qui restera-t-il sur terre sinon des profiteurs, des flatteurs, des êtres malfaisants, dépourvus d'humanité. Le rêve fou d'une victoire sur le temps se solde par le triomphe du mal à la surface de la terre. Et on peut se demander si un être comme Pobedonosikov n'est pas une araignée vivante, embusquée dans les couloirs sombres de la société socialiste.

Sous le masque du jeu, de la féerie, des marionnettes, des feux d'artifice, la pièce de Majakovskij revêt un contenu désespéré. Son caractère festif, redoublé, à sa création, par la mise en scène distanciée de Mejerkhol'd, laisse percer une note de désespoir. Et si nous, les spectateurs, nous nous retrouvions enfermés dans les entrailles mystérieuses de la banja présente-absente ? Dans cette tentative insensée pour s'arracher au temps et à l'espace nous sommes confrontés au tragique d'une existence vouée à l'absurde, cet absurde qui anime une société en pleine dérive politique et idéologique. Ne peut-on considérer Banja comme une pièce existentialiste avant la lettre, mais en filigrane et en contrebande.

(C) Gérard Abensour

Professeur émérite INALCO

22 Charles Baudelaire, Spleen, Euvres complètes, Paris, Gallimard, La Pléiade, 1961, p.71. (Première édition en 1857. Dostoievski n'a-t-il pas eu connaissance des poésies de Baudelaire ?) 


\section{Résumés}

On trouve au moins trois traductions différentes de Banja, titre de la pièce de Majakovskij : Les Bains, La Grande lessive, Les étuves. Comment rendre le sens de cette institution rurale mystérieuse ? Lieu de purification, de renaissance, de naissance et de mort, abri d'un esprit malfaisant. A la suite de Khlebnikov, qui meurt dans une banja, Majakovskij , domestique le temps, pour dominer le présent. Sa Femme phosphorescente vient, comme dans l'Apocalypse pour sauver les Justes et punir les méchants. II cache son angoisse existentielle derrière le rire, le burlesque et la satire. Satire contre les dignitaires soviétiques qui précède de peu son suicide en 1930.

It is very hard to translate in French Banja, the title of Majakovskij 's play. In fact banja is a rural institution with a contradictory meaning: it is a place where people wash themselves but it is also a place of purification of the soul and the residence of a small devil. Making an echo to Khlebnikov, Majakovskij acts on time, going forward to the future, he appeals to the Apocalypsis (The phosphorescent Woman reminds the Woman with a crown of stars ). He hides his mysticism under laugh, burlesque and satire. Under the cover of his criticism of Soviet dignitaries Majakovskij hides his deep anguish. The end will be his suicide a month after the opening of the play in 1930.

Маяковский так писал о своей последней пьесе: "Что такое Баня и кого она моет?" Ясно, что "баня"--это метафора, в "банях", термах, души грешников должны очиститься от грязи и пороков. Там встречаются полярные понятия, чистота души и грязь дьявольских сил. Маяковский следует за Хлебниковым: он подчинает себе время, он вводит Фосфорическую Женщину, "Жену, облаченную в солнце" из Апокалипсиса. Свою глубокую личную и социальную тревогу Маяковский скрывает под гротеском, фейерверком, сценическими эффектами: он покончит с собой ровно через месяц после премьеры Бани.

\section{Note biographique :}

Gérard Abensour, professeur émérite de langue et littérature russe, INALCO, ENS Lyon. Nombreux articles sur le théâtre russe. Vsévolod Meyerhold ou l'invention de la mise en scène, Paris, Fayard, 1998. Site : <http://gerard-abensour.fr $>$ 